\title{
Research on Second Language Acquisition from the Perspective of Dynamic System Theory
}

\author{
Xianwu Sun \\ School of Foreign Languages, Northwest Minzu University, Lanzhou, 730030, China
}

Keywords: Second language acquisition, Dynamic system theory, Dynamic interaction

\begin{abstract}
Dynamic system theory has provided a new research perspective for the study of second language acquisition, which provides the new interpretation for the problems in the traditional second language acquisition. This paper discusses the basic principles and core concepts of dynamic system theory, analyzes the dynamic system theory and interlanguage fossilization in the second language acquisition process, the relations between language variation and environment as well as other factors, and provides reference for related researchers.
\end{abstract}

\section{Introduction}

Dynamic system theory stems from applied mathematics, which is the theory of dynamics process about system status transfer, and its central topic is to master the evolution rule of the system. In 1997, Lassen Freeman firstly applied it into second language acquisition. System science always has numerous similarities with second language acquisition, such as complex non-linear system. They always focus on the transition from disorder to order. Therefore, it can attempt to apply the dynamic system theory to second language acquisition. Under this theoretical frame, language is regarded as a dynamic system, and language development is a dynamic process. As a sub-system, language learners are also a dynamic system, consisting of memory, attention, motive and other secondary sub-systems. In the process of second language acquisition, while making progress continuously, learners may experience degeneration or corrosion in some period. Namely, language study is not a process of linear increase, but a process of repeated fluctuation. If the whole process of language study is taken as a system, each element composing the language is the component of this system. Although numerous non-linear scholars point out that each element in the study system is not the simple causal relations, the lack of theories makes researchers had to explain the non-linear characteristics of language study process and the unpredictability of study results. Therefore, dynamic system theory is locally introduced into the study of second language acquisition, used to interpret the complex and dynamic process of second language acquisition. Dynamic system theory melts the recognition and society views of language development as one, so as to become the method to solve the coherence of various second language problem. As the emerging theory of second language acquisition research field, dynamic system theory mainly focuses on theoretical discussion in the early period. In recent years, it began developing related empirical study, and the quantity and research topic is limited. The lack of empirical study has become the carrier for the further development and application of this theory. It is also the truth in our country. This paper teases the concepts and problems that mostly relate to dynamic system theory and second language acquisition, melts dynamic system theory in the second language acquisition for research and analysis, and displays the application potentials of this theory in second language field.

\section{Basic Principles and Core Concepts of Dynamic System Theory}

Basic Principles. Dynamic system theory is also called as complex theory, non-linear self-organization system theory. It emphasizes the importance of time relations and the relationship between each element in the system. Dynamic system theory stems from Newton mechanics. Like other nature science and engineering rule, this theory attempts to find out the relations between the 
status of the system at this moment and the future status, and discover the mutual function and effects between each variable inside the system. In dynamic system theory, it is believed that the system has the complex internal relevance, and its internal variables connect with each other. It will cause effects on all other variables inside the system. The overall behavior and reflection of the complex system stems from the interaction between all internal sub-systems and components and the interaction between the system and the external environment. Because internal and external system factors function and change continuously, the system has never been at the static status, and it is quite difficult to predict the ultimate status of the system.

Different from traditional linear development views, the development process is all non-linear in the perspective of dynamic system theory, which may exist degeneration or corrosion. Under the dynamic system theory frame, second language acquisition study breaks through the traditional linear, sweeping and reductionism normal forms, which turns to focus on the variation reflected by large amounts of changing details in the second language acquisition process, as well as the complex mutual function between system factors in the second language acquisition system reflected by these variations.

Therefore, dynamic system theory oriented second language acquisition research should focus on the empirical study above the theoretical discussion, striving to find out the complex association and mutual function in the second language system development process. However, the current domestic and foreign empirical study is just started, and the domestic related studies are mostly at the unitary theoretical level, empirical study is rare. Although it has no relevance to the abstractness of dynamic system theory itself, it should attribute to the lack of research methods and analytic tools under this theory more importantly. It teases the empirical study of second language acquisition under the dynamic system theory, exploits the feasible and practical research methods and analytic tools, and becomes an important mission.

Core Concepts. The dynamic system theory of language has three groups of principles as below: dynamic, non-linear, muddy, unpredictable: complex, open, self-organization, self-adaptability; sensitive dependence on initial conditions and sensitive dependence on feedback. Among them, several basic concepts are the core part of dynamic system theory.

Non-linearity. It means that in this system, results and reasons are not proportional, namely reasons will not definitely lead to results. It is different from the belief of the traditional linear system that the reason of forming some force will cause the equal force of results. For example, the long-term efforts of study by language learners will not definitely acquire the excellent language ability. However, accidental events, such as going abroad, can activate the motive and strong study will of learners, so as to improve the foreign language level.

Self-organization language will respond to external input, interact with internal sub-system, and cause the system recombination through self-organization. Language system recombines itself continuously and it never stops.

Sensitive dependence on initial conditions, called as butterfly effect as well. Any tiny change in the system, even the accidental random change will affect the whole body and causes an unpredictable result. It demonstrates the unpredictability of language development. Once dynamics equation is determined, the behaviors of dynamics system, namely its motion and variation, are confirmed by original conditions; if original conditions are similar, the behaviors of dynamics system are also drawing near. This decision theory creed makes the important function on numerous routine scientific problems such as celestial operation, object motion, etc. However, some non-linear dynamic system shows the obvious deviation.

Variation refers to the variation of learners on pronunciation, vocabulary, sentence building and grammar. It stems from the self-organization in the system. Because every learner follows the orbit self-organization development, variation is the necessary component of any system, which is inevitable.

Non-rebuilt balance state. Language development system essentially has the innovation and self-organization, which accompanies with normal attractors at the same time. With the time passing, attractors become more stable and anti-interference. 


\section{Second Language Acquisition Research Contents under Dynamic System Theory}

Dynamic System Theory and Interlanguage Fossilization. In the entire second language study process, except for interacting with external factors, they will also organize their own language system on their own. Due to the self-organizing property of second language system, the system usually experiences two statuses, namely absorption and repulsion form. The former indicates that second language system is at the stagnated status from the surface, and the latter means that the system is at the turbulence.

When the system is at the absorption form, learners will be trapped into a stagnated difficulty. At this time, learners need to adjust and complete the breakthrough, and the fossilization phenomenon of interlanguage is a typical absorption form. The traditional concept of interlanguage fossilization is a kind of final result status. Under the perspectives of dynamic system theory, fossilization is a status that the system becomes easy to stay when compared to other statuses, which can be only overcome by the powerful force as a temporary status. Fossilization phenomenon is not only a recognition phenomenon, but also a social phenomenon. While describing and explaining the fossilization phenomenon of language development termination status, previous researchers ignore a fact that the language in use is continuously developing and changing. With the continuous use and development of language, fossilization phenomenon is the unpredictable result caused by the mutual effects of various variables continuously inside learners, which is a reflection of "potential boundlessness" instead of the termination status of language study, because the system status starts exercising again at the next moment, recombine and develop continuously.

From here, we can see the difference of two concepts: the traditional fossilization is a kind of final status, which will not change any more, but the absorption state is temporary. From the angle of language teaching, absorption state should not be taken as the final status, but we should see "the infinity of potentials”. It is no doubt that it has positive meaning to language teaching.

Dynamic System Theory and Language Variation. Variation refers to the difference of speakers in the pronunciation, grammar or vocabulary selection of a language, and the study of variation is an important topic of second language acquisition research. The data of second language learners usually shows huge differences. Previous researchers often attempt explaining free variation to the system with the complete variation settings in the data of learners. The systematization of language internal system is used to conceal in a black box that triggers language structure, and this language structure shows the linear distribution.

Traditional second language acquisition opinion uses linear methods to explain various language problems and behavioral problems of learners. It is believed that individual variation is background noise is often regarded as extreme value or measurement error can be ignored. From the angle of dynamic system theory, researchers have altered the conclusion of language acquisition's linear development, and morpheme acquisition sequence's variation all exist between learners themselves and individuals. Individual variation should not be taken as the stable and fixed individual characteristic, and language development is the prelude from one mark to another, reflecting the discrete development of language. From the frequency, topic of language input, emotional factors of learners, foreign language aptitude and motives, researchers describe the effects of individual differences on language development that cannot be ignored.

Only by melting these seemingly contradictory factors can we find various complex dynamic factors behind the phenomenon. Therefore, language variation is a key and necessary step for second language acquisition development process to understand the dynamic system, and observing individual variation from the angle of dynamic system theory can help us find the language development modes more comprehensively.

Dynamic System Theory and Study Environment. Larsen Freeman (1997) believed that language study is process of building meaningful language dynamic communication mode with the aid of teaching materials and other language materials through the guidance of teachers. Larsen Freemen and Cameron put forward the mutual function between the language resources of learners and surrounding environment in the process of second language development. As the independent system, learners and language environment adjust and affect each other while developing 
dynamically on their own, so as to reach the relative balance. Language study is not only to study abstract symbols in isolation, but also apply them into the reality life in the end. It reflects the dynamic communication scenario.

For example, after learning language knowledge, learners should communicate with the external system, such as environment, teaching materials or others to really master language knowledge. If they ignore the dynamic communication with the context and only stay in the internal digestion of individual system, even if they remember at one time, their memory cannot last long and promote the constant development of language. It is the reason of the phenomenon why learners always cannot remember words. While studying the second language in the bilingual environment, learners have the chance to touch more target languages through various mediums. These target languages not only include more complex and diversified target language forms, but also display in the multiple modes.

As a result, the input volume and language state of second language in the bilingual environment is obviously more than the mono-lingual. Instead, multiple language forms that appear repeatedly can not only satisfy the choices of different levels of learners, but also promote the formation of language automation. In addition, multi-mode input can not only deepen the impression of language forms, but also lower the fatigue of study and enhance the study interest. One of effective methods to compensate the defects of monolingual environment is to increase the input volume of extracurricular second language and develop multiple language activities. In class environment, it expands the effective language input for learners as much as possible, improve the input efficiency of key contents, provide more chances of language interaction, and enhance the guidance on language forms. In extracurricular environment, it provides the bilingual environment in each place as much as possible, fully use online multimedia, and make learners have more chances to touch multi-mode target languages.

\section{Conclusion}

Compared to traditional second language acquisition study, second language acquisition study in the dynamic system theory vision of field adopts wholly new opinions and methods. It abandons traditional study methods based on linear causal relations, adopts multiple factors of dynamic interactive opinions, focuses on the dynamic development of second language acquisition in different levels and periods, so as to be more proper for the description of second language acquisition. Second language acquisition study based on dynamic system theory can also provide very benign reference for foreign language teaching.

\section{References}

[1] Zhang Zhenhong, Yang Xiaoming. Review of Empirical Studies of Second Language Acquisition within the Framework of Dynamic System Theory [J]. Journal of Tianjin University (Social Sciences), 2015, 17(2): 156-161.

[2] Xu Jinfen, Lei Pengfei. A study on classroom second language acquisition fromdynamic system theory perspective: theoreticframework and research approaches [J].Foreign Language Learning Theory and Practice, 2017(1): 22-29+9.

[3] Dai Yuncai, Wang Tongshun. Constructing a Dynamic Model of SLA Based on the Interplay of Environment, Learner and Language [J].Shandong Foreign Language Teaching Journal, 2012(5): 36-42.

[4] Li Mengli, Fan Lin. Connectionism and Dynamic System theory in SLA Research [J]. Journal of Ningbo University (Liberal Arts Edition), 2016, 29(2): 38-42.

[5] Deng Xinghua. An Overview of Second Language Acquisition from thePerspective of Dynamic System Theory [J].Journal of HechiUniversity, 2014, 34(3): 39-45. 